\title{
Optimal advertising and pricing decisions for complementary products
}

\author{
Ata Allah Taleizadeh • Masoud Charmchi
}

Received: 22 September 2014/ Accepted: 12 January 2015/Published online: 5 February 2015

(C) The Author(s) 2015. This article is published with open access at Springerlink.com

\begin{abstract}
Cooperative advertising is an agreement between a manufacturer and a retailer to share advertising cost at the local level. Previous studies have not investigated cooperative advertising for complementary products and their main focus was only on one good. In this paper, we study a two-echelon supply chain consisting of one manufacturer and one retailer with two complementary goods. The demand of each good is influenced not only by its price but also by the price of the other product. We use two game theory approaches to model this problem; Stackelberg manufacturer and Stackelberg retailer.
\end{abstract}

Keywords Pricing - Advertising - Game theory - Supply chain $\cdot$ Complementary $\cdot$ Stackelberg

\section{Introduction}

In retailing, cooperative advertising refers to an agreement between a manufacturer and a retailer whereby the manufacturer will reimburse the retailer in part or in full for advertising expenditures. In a two-echelon supply chain, the relationship between a manufacturer and a retailer may be either non-cooperative or cooperative. In non-cooperative models, the party who has more power plays as a leader and the other part is a follower. The leader is the part who decides the first move and anticipates the follower's move (Gaski 1984; Munson and Rosenblatt 2001).

A. A. Taleizadeh $(\bowtie) \cdot$ M. Charmchi

School of Industrial Engineering, College of Engineering, University of Tehran, Tehran, Iran

e-mail: Taleizadeh@ut.ac.ir

M. Charmchi

e-mail: masoud.charmchi@ut.ac.ir
In this paper, we seek to analyze a two-echelon supply chain comprising of a manufacturer and a retailer with two complementary goods. The demand of each product is influenced not only by its price but also by the price of the other good. The demand for computer and its software is an example in this regard. Another example of these products is Torch and battery. In spite of previous studies, this paper investigates the advertising and pricing decisions for two complementary products where the price of each product affects the demand of the other. To model the problem, two game theory models are developed including Stackelberg manufacturer and Stackelberg retailer.

Pricing as an invaluable tool is studied by many researchers. Taleizadeh and Noori-Daryan (2015) studied the pricing, inventory and production decisions in a threelevel supply chain. Then, Taleizadeh et al. (2015a) developed previous work by considering rework process and buyback of scraped item Taleizadeh et al. (2015b).

Many papers studied cooperative advertising and pricing decisions in a two-level supply chain. Huang and Li (2001) studied cooperative advertising between one manufacturer and one retailer. They did not consider price deduction in their models. They discussed three models, two noncooperative games and one cooperative game. Yue et al. (2006a, b) considered cooperative advertising in a price sensitive market. They added price deduction to the previous models and showed that considering price deduction enhances the total profit of the supply chain. Szmerekovsky and Zhang (2009) evaluated pricing and two-tier advertising model for single product where the manufacturer plays as the leader and the retailer acts as the follower in a Stackelberg game. They showed that when the retailer advertising is inefficient, it is better for the manufacturer to decrease the wholesale price instead of investing on advertising in the retailer level. Xie and Neyret (2009) 
developed four different models consisting of three noncooperative games (Nash, Stackelberg retailer and Stackelberg manufacturer) and one cooperative game. They showed that the leader of the game always prefers to make the follower invest more in advertisement. Jørgensen et al. (2000) distinguished between the retailer's and the manufacturer's advertising expenditures and used an advertising function that was influenced by both types of advertising expenditures. Just like what is seen in (Xie and Wei 2009; Huang et al. 2002). SeyedEsfahani et al. (2011) developed a model in which the relationship between price and demand had a relatively general form in comparison with the classic linear relationship. The customer's demand was influenced by advertising and price expenditures. They demonstrated that the shape of the demand price function may change the optimal values of decision variables and channel members profit. Aust and Buscher (2012) used another approach to solve the models in which vertical cooperative advertising is used between the members of a chain. Hosseini et al. (2013) developed a multiple objective approach for joint ordering and pricing planning problem with stochastic lead times.

There are some papers that studied complementary products without considering cooperative advertising (Gabszewicz et al. 2001). Yan and Bandyopadhyay (2011) studied two complementary products and showed that when the degree of complement between two products is high, it is better to use bundling policy. Yue et al. (2006a, b) considered a supply chain consisting of two complementary products and the customer needed to buy these complementary products as a mixed bundle. Yan et al. (2014) developed a model with two complementary products. In their models, marketing cost was embedded into the model. Wei et al. (2013) studied complementary products in two-echelon supply chain. They developed five models, the MS-Stackelberg, MS-Bertrand, RS-Stackelberg, RS-Bertrand and Nash. They did not consider the effect of advertising expenditure in their models.

In this article, we consider a two-echelon supply chain consisting of one manufacturer and one retailer with two complementary products. The market demands of both goods are influenced by price and advertising. We develop two models, under Stackelberg-manufacturer and Stackelberg-retailer strategies.

\section{Problem definition and modeling}

We study cooperative advertising for complementary products in a two-echelon supply chain consisting of one manufacturer and one retailer. Complementary products are those products that are used together to satisfy particular requirement, therefore the demand of each product decreases as the price of another product increases. To define the problem, we introduce notations in Table 1.

According to Table 1, decision variables for the manufacturer and the retailer are their prices $\left(w_{1}, w_{2}, p_{1}, p_{2}\right)$, advertising expenditures $\left(q_{m}, q_{r}\right)$ and the manufacturer's participation rate $(t)$. Regarding the previous studies (Xie and Wei 2009; Xie and Neyret 2009; SeyedEsfahani et al. 2011), we assume that the consumer's demand for both products $\left[D_{i}\left(P_{1}, P_{2}, q_{r}, q_{m}\right)\right]$ depends on price of products $\left(P_{1}, P_{2}\right)$ and advertising expenditure $\left(q_{m}, q_{r}\right)$ and has the following form:

$$
\begin{aligned}
D_{i}\left(P_{1}, P_{2}, q_{r}, q_{m}\right) & =M\left(q_{m}, q_{r}\right) \cdot R_{i}\left(P_{1}, P_{2}\right) \\
i & =1,2
\end{aligned}
$$

The impact of advertising expenditure on the consumer demand $\left[M\left(q_{m}, q_{r}\right)\right]$ is equal for both products and both types of efficacies of advertising could influence it (Jørgensen et al. 2000; Huang et al. 2002; Xie and Wei 2009), which is shown in Eq. (2).

$M\left(q_{r}, q_{m}\right)=\left[k_{r} \sqrt{q_{r}}+k_{m} \sqrt{q_{m}}\right]$

Since the demand for given good varies inversely with the price of complementary goods, we use a similar approach to Yan and Bandyopadhyay (2011) such that:

$R_{1}\left(P_{1}, P_{2}\right)=\left(1-\beta p_{1}-\beta \theta p_{2}\right)$

$R_{2}\left(P_{1}, P_{2}\right)=\left(1-\beta p_{2}-\beta \theta p_{1}\right)$

and the following restricts ensure that $R_{i}\left(P_{1}, P_{2}\right)$ are always positive:

$$
\begin{aligned}
& \left(1-\beta p_{1}-\beta \theta p_{2}\right)>0 \Rightarrow p_{1}+\theta p_{2}<\frac{1}{\beta} \\
& \left(1-\beta p_{2}-\beta \theta p_{1}\right)>0 \Rightarrow p_{2}+\theta p_{1}<\frac{1}{\beta}
\end{aligned}
$$

Using Eqs. (2), (3) and (4), we have:

$$
D_{1}\left(p_{1}, p_{2}, q_{r}, q_{m}\right)=\left(1-\beta p_{1}-\beta \theta p_{2}\right)\left[k_{r} \sqrt{q_{r}}+k_{m} \sqrt{q_{m}}\right]
$$

$D_{2}\left(p_{1}, p_{2}, q_{r}, q_{m}\right)=\left(1-\beta p_{2}-\beta \theta p_{1}\right)\left[k_{r} \sqrt{q_{r}}+k_{m} \sqrt{q_{m}}\right]$

Therefore, the manufacturer, the retailer and the system profits are formulated as:

$$
\begin{aligned}
\pi_{m}=[ & \left.k_{r} \sqrt{q_{r}}+k_{m} \sqrt{q_{m}}\right]\left[\left(w_{1}-d_{1}\right)\left(1-\beta p_{1}-\beta \theta p_{2}\right)\right. \\
& \left.+\left(w_{2}-d_{2}\right)\left(1-\beta p_{2}-\beta \theta p_{1}\right)\right]-t q_{r}-q_{m}
\end{aligned}
$$

$$
\begin{aligned}
\pi_{r}=[ & \left.k_{r} \sqrt{q_{r}}+k_{m} \sqrt{q_{m}}\right]\left[\left(p_{1}-w_{1}\right)\left(1-\beta p_{1}-\beta \theta p_{2}\right)\right. \\
& \left.+\left(p_{2}-w_{2}\right)\left(1-\beta p_{2}-\beta \theta p_{1}\right)\right]-(1-t) q_{r}
\end{aligned}
$$




\section{Table 1 Notations}

\begin{tabular}{ll}
\hline$d_{i}$ & Unit manufacturing cost of products $i$ where $i=1,2$ \\
$\theta$ & The complementary degree between two products \\
$K_{r}$ & The efficacy of retailer's advertising in generating sales \\
$K_{m}$ & The efficacy of manufacturer advertising in generating sales \\
$\beta$ & The sensitivity of demand rate respect to price \\
$p_{i}$ & Unit selling price of product $i$ where $i=1,2$, (retailer's \\
& $\quad$ decision variables) \\
$W_{i}$ & Unit wholesale price of product $i$ where $i=1,2$, \\
& (manufacturer's decision variables) \\
$q_{r}$ & Retailer's local advertising expenditure, (retailer's decision \\
& variable) \\
$q_{m}$ & Manufacturer's national advertising expenditure \\
& (manufacturer's decision variable) \\
$t$ & Manufacturer participation rate \\
$\pi_{m}$ & Profit function of the manufacturer \\
$\pi_{r}$ & Profit function of the retailer \\
$\pi_{m+r}$ & Profit function of the system
\end{tabular}

$$
\begin{aligned}
\pi_{m+r}=[ & \left.k_{r} \sqrt{q_{r}}+k_{m} \sqrt{q_{m}}\right]\left[\left(p_{1}-c_{1}\right)\left(1-\beta p_{1}-\beta \theta p_{2}\right)\right. \\
& \left.+\left(p_{2}-c_{2}\right)\left(1-\beta p_{2}-\beta \theta p_{1}\right)\right]-q_{r}-q_{m}
\end{aligned}
$$

$$
\begin{array}{ll}
\text { St: } & p_{1}+\theta p_{2}<\frac{1}{\beta}, \quad p_{2}+\theta p_{1}<\frac{1}{\beta} \\
& 0<w_{1}<p_{1}, \quad 0<w_{2}<p_{2}, 0 \leq t \leq 1 \text { and } 0<q_{r}, q_{m}
\end{array}
$$

\section{Two games model}

\section{Stackelberg manufacturer}

In this section, we model the problem as a non-cooperative game. We consider the manufacturer as the leader and the retailer as the follower in the Stackelberg equilibrium game theory. Manufacturer takes the action first and then the retailer moves sequentially. To find the solution, we first solve the retailer's problem as follows:

$$
\begin{array}{r}
\pi_{r}=\left[k_{r} \sqrt{q_{r}}+k_{m} \sqrt{q_{m}}\right]\left[\left(p_{1}-w_{1}\right)\left(1-\beta p_{1}-\beta \theta p_{2}\right)\right. \\
\left.+\left(p_{2}-w_{2}\right)\left(1-\beta p_{2}-\beta \theta p_{1}\right)\right]-(1-t) q_{r}
\end{array}
$$

$\pi_{r}$ is a concave function with respect to retailer's decision variables so we can solve the problem as follows:

$$
\begin{aligned}
\frac{\partial \pi_{r}}{\partial p_{1}}=0 \Rightarrow p_{1} & =\left(\frac{1+\beta w_{1}(1+\theta)}{2 \beta(1+\theta)}\right) \\
\frac{\partial \pi_{r}}{\partial p_{2}}=0 \Rightarrow p_{2} & =\left(\frac{1+\beta w_{2}(1+\theta)}{2 \beta(1+\theta)}\right) \\
\frac{\partial \pi_{r}}{\partial q_{r}}=0 \Rightarrow q_{r}= & \left(\frac{k_{r}}{2(1-t)}\right)^{2}\left[\left(\frac{1-\beta w_{1}(1+\theta)}{2 \beta(1+\theta)}\right)\right. \\
& \times\left(\frac{\left(1-\beta w_{1}-\beta w_{2} \theta\right)}{2}\right)+\left(\frac{1-\beta w_{2}(1+\theta)}{2 \beta(1+\theta)}\right) \\
& \left.\times\left(\frac{\left(1-\beta w_{2}-\beta w_{1} \theta\right)}{2}\right)\right]^{2}
\end{aligned}
$$

Using Eqs. (13) and (14), retailer's price decreases, as $\theta$ increases and increases as the manufacturer's wholesale price of each product increases. We can understand from Eq. (15) that optimal local advertising level decreases as the complement degree between two products $(\theta)$ increases. When the manufacturer participation rate $(t)$ decreases, the retailer's local advertising level $\left(q_{r}\right)$ decreases as well.

Now, we seek to solve the manufacturer's problem.

$$
\begin{aligned}
\pi_{m}= & {\left[k_{r} \sqrt{q_{r}}+k_{m} \sqrt{q_{m}}\right]\left[\left(w_{1}-d_{1}\right)\left(1-\beta p_{1}-\beta \theta p_{2}\right)\right.} \\
& \left.+\left(w_{2}-d_{2}\right)\left(1-\beta p_{2}-\beta \theta p_{1}\right)\right]-t q_{r}-q_{m}
\end{aligned}
$$

Substituting $p_{1}, p_{2}$ and $q_{r}$ from Eqs. (13), (14), (15) and setting the first derivatives of profit functions with respect to the decisions variables, equal to zero, yields:

$$
\begin{aligned}
\frac{\partial \pi_{m}}{\partial w_{1}} & =0 \Rightarrow \frac{\left[\left(w_{1}-d_{1}\right)\left(1-\beta w_{1}-\beta w_{2} \theta\right)+\left(w_{2}-d_{2}\right)\left(1-\beta w_{2}-\beta w_{1} \theta\right)\right]}{\left[\left(1-\beta w_{1}(1+\theta)\right)\left(1-\beta w_{1}-\beta w_{2} \theta\right)+\left(1-\beta w_{2}(1+\theta)\right)\left(1-\beta w_{2}-\beta w_{1} \theta\right)\right]} \\
& =\frac{k_{r}^{2}\left[1-\beta\left(3 w_{1}+3 w_{2} \theta\right)+2 \beta\left(d_{1}+\theta d_{2}\right)\right]}{4 \beta(1+\theta)\left[\beta w_{1}\left(3 k_{r}^{2}+4 k_{m}^{2}\right)+\beta w_{2} \theta\left(3 k_{r}^{2}+4 k_{m}^{2}\right)-\left(k_{r}^{2}+2 k_{m}^{2}\right)-2 \beta\left(d_{1}+\theta d_{2}\right)\left(k_{r}^{2}+k_{m}^{2}\right)\right]} \\
\frac{\partial \pi_{m}}{\partial w_{2}} & =0 \Rightarrow \frac{\left[\left(w_{1}-d_{1}\right)\left(1-\beta w_{1}-v \beta w_{2} \theta\right)+\left(w_{2}-d_{2}\right)\left(1-\beta w_{2}-\beta w_{1} \theta\right)\right]}{\left[\left(1-\beta w_{1}(1+\theta)\right)\left(1-\beta w_{1}-\beta w_{2} \theta\right)+\left(1-\beta w_{2}(1+\theta)\right)\left(1-\beta w_{2}-\beta w_{1} \theta\right)\right]} \\
& =\frac{k_{r}^{2}\left[1-\beta\left(3 w_{2}+3 w_{1} \theta\right)+2 \beta\left(d_{2}+\theta d_{1}\right)\right]}{4 \beta(1+\theta)\left[\beta w_{2}\left(3 k_{r}^{2}+4 k_{m}^{2}\right)+\beta w_{1} \theta\left(3 k_{r}^{2}+4 k_{m}^{2}\right)-\left(k_{r}^{2}+2 k_{m}^{2}\right)-2 \beta\left(d_{2}+\theta d_{1}\right)\left(k_{r}^{2}+k_{m}^{2}\right)\right]}
\end{aligned}
$$


By solving Eqs. (17) and (18), optimal values of $w_{1}$ and $w_{2}$ and the other variables are obtained as follows (The proof is provided in "Appendix A"):

$$
\begin{aligned}
q_{m}= & \left(\frac{k_{m}^{2}}{16}\right)\left[\left(w_{1}^{*}-d_{1}\right)\left(1-\beta w_{1}^{*}-\beta w_{2}^{*} \theta\right)\right. \\
& \left.+\left(w_{2}^{*}-d_{2}\right)\left(1-\beta w_{2}^{*}-\beta w_{1}^{*} \theta\right)\right]^{2} \\
t^{*}= & \left(\frac{z_{2}^{*}(8 \beta(1+\theta))-z_{1}^{*}}{z_{2}^{*}(8 \beta(1+\theta))+z_{1}^{*}}\right) \\
p_{1}^{*}= & \left(\frac{1+\beta w_{1}^{*}(1+\theta)}{2 \beta(1+\theta)}\right) \\
p_{2}^{*}= & \left(\frac{1+\beta w_{2}^{*}(1+\theta)}{2 \beta(1+\theta)}\right) \\
q_{m}^{*}= & \left(\frac{k_{r}}{2\left(1-t^{*}\right)}\right)^{2}\left[\left(\frac{1-\beta w_{1}^{*}(1+\theta)}{2 \beta(1+\theta)}\right)\right. \\
& \times\left(\frac{\left(1-\beta w_{1}^{*}-\beta w_{2}^{*} \theta\right)}{2}\right)+\left(\frac{1-\beta w_{2}^{*}(1+\theta)}{2 \beta(1+\theta)}\right) \\
& \left.\times\left(\frac{\left(1-\beta w_{2}^{*}-\beta w_{1}^{*} \theta\right)}{2}\right)\right]^{2}
\end{aligned}
$$

where $z_{1}^{*}$ and $z_{2}^{*}$ are defined in ("Appendix A").

Stackelberg retailer

In this section, the retailer takes on more power and plays the leadership role. The solution of this problem is called SR equilibrium. We first solve the manufacturer's problem as follows:

$$
\begin{aligned}
\pi_{m}= & {\left[k_{r} \sqrt{q_{r}}+k_{m} \sqrt{q_{m}}\right]\left[\left(w_{1}-d_{1}\right)\left(1-\beta p_{1}-\beta \theta p_{2}\right)\right.} \\
& \left.+\left(w_{2}-d_{2}\right)\left(1-\beta p_{2}-\beta \theta p_{1}\right)\right]-t q_{r}-q_{m}
\end{aligned}
$$

St: $0 \leq t \leq 1,0<q_{m}, w_{1}<p_{1}$ and $w_{2}<p_{2}$

Since $\pi_{m}$ is a decreasing function of $t$, the optimal value of $t$ is zero. $\pi_{m}$ is an increasing function of $w_{1}$ and $w_{2}$ which means that the optimal values of $w_{1}$ and $w_{2}$ are $p_{1}$ and $p_{2}$, respectively, and lead to $\pi_{r}=0$. To avoid this, we use a similar approach as Jørgensen and Zaccour (1999), Xie and Neyret (2009) have used. According to their works, the retailer's margins for both goods are formulated as:

$p_{1}-w_{1}=w_{1}-d_{1} \Rightarrow w_{1}=\frac{p_{1}+d_{1}}{2}$

$p_{2}-w_{2}=w_{2}-d_{2} \Rightarrow w_{2}=\frac{p_{2}+d_{2}}{2}$

By substituting Eqs. (24) and (25) into the manufacturer's problem and solving it, we can reach the manufacturer's decision variables as follows (See "Appendix B”):

$$
\begin{aligned}
w_{1}^{*}= & \frac{1+3 \beta d_{1}(1+\theta)}{4 \beta(1+\theta)} \\
w_{2}^{*}= & \frac{1+3 \beta d_{2}(1+\theta)}{4 \beta(1+\theta)} \\
q_{m}= & \left(\frac{k_{m}}{2}\right)^{2}\left[( w _ { 1 } - d _ { 1 } ) \left(1-\beta 2 w_{1}+\beta d_{1}-\beta \theta 2 w_{2}\right.\right. \\
& \left.+\beta \theta d_{2}\right)+\left(w_{2}-d_{2}\right)\left(1-\beta 2 w_{2}+\beta d_{2}\right. \\
& \left.\left.-\beta \theta 2 w_{1}-\beta \theta d_{1}\right)\right]^{2}
\end{aligned}
$$

$t^{*}=0$

By substituting Eqs. (26), (27) into (24) and (25), we have:

$p_{1}^{*}=\frac{1+\beta d_{1}(1+\theta)}{2 \beta(1+\theta)}$

$p_{2}^{*}=\frac{1+\beta d_{2}(1+\theta)}{2 \beta(1+\theta)}$

By substituting Eqs. (26), (27) and (28) into the retailer's problem and setting $\partial \pi / \partial q_{r}$ equal to zero, we have:

$$
\begin{aligned}
\frac{\partial \pi_{r}}{\partial q_{r}}=0 \rightarrow q_{r}^{*}= & \left(\frac{k_{r}}{2}\right)^{2}\left[\left(p_{1}^{*}-w_{1}^{*}\right)\left(1-\beta p_{1}^{*}-\beta \theta p_{2}^{*}\right)\right. \\
& \left.+\left(p_{2}^{*}-w_{2}^{*}\right)\left(1-\beta p_{2}^{*}-\beta \theta p_{1}^{*}\right)\right]^{2}
\end{aligned}
$$

\section{Numerical examples and discussion of the results}

In this section, we compare the results of models through some examples. In Table 2, the value of the decision variables for different values of $\theta$ in SM model is shown. In this example, we have $d_{1}=6, d_{2}=8, k_{m}=0.7, k_{r}=$ 0.4 and $\beta=0.06$.

Table 2 indicates that when the complementary degree between two products is large, it is not optimal for the manufacturer and the retailer to advertise, and their profits decrease as $\theta$ increases.

From Fig. 1, when the manufacturer plays as the leader, his profit is greater than retailer and the profit of manufacturer, retailer and whole system decreases as the complement degree between two products $(\theta)$ increases. For large values of $\theta$, it is better for the manufacturer and the retailer to use bundling strategy to increase their profits (Table 3 ).

From Fig. 2, when the retailer plays as a leader, his profit is greater than manufacturer and the profit of the manufacturer, the retailer and whole system decrease as the degree of complementary between the two products $(\theta)$ increases. 
Table 2 Impact of $\theta$ on variables of SM game

\begin{tabular}{lccccl}
\hline$\theta$ & 0.2 & 0.3 & 0.4 & 0.5 & 0.6 \\
\hline$w_{1}^{*}$ & 9.62 & 9.13 & 8.71 & 8.35 & 8.03 \\
$w_{2}^{*}$ & 10.70 & 10.21 & 9.79 & 9.43 & 9.11 \\
$p_{1}^{*}$ & 11.75 & 10.97 & 10.31 & 9.73 & 9.22 \\
$p_{2}^{*}$ & 12.29 & 11.51 & 10.85 & 10.27 & 9.76 \\
$q_{r}^{*}$ & 0.049 & 0.029 & 0.017 & 0.009 & 0.005 \\
$q_{m}^{*}$ & 0.090 & 0.054 & 0.031 & 0.018 & 0.009 \\
$t^{*}$ & 0.5460 & 0.5460 & 0.5460 & 0.5460 & 0.5460 \\
$\pi_{m}^{*}$ & 0.140 & 0.084 & 0.049 & 0.028 & 0.015 \\
$\pi_{r}^{*}$ & 0.129 & 0.077 & 0.045 & 0.025 & 0.014 \\
\hline
\end{tabular}

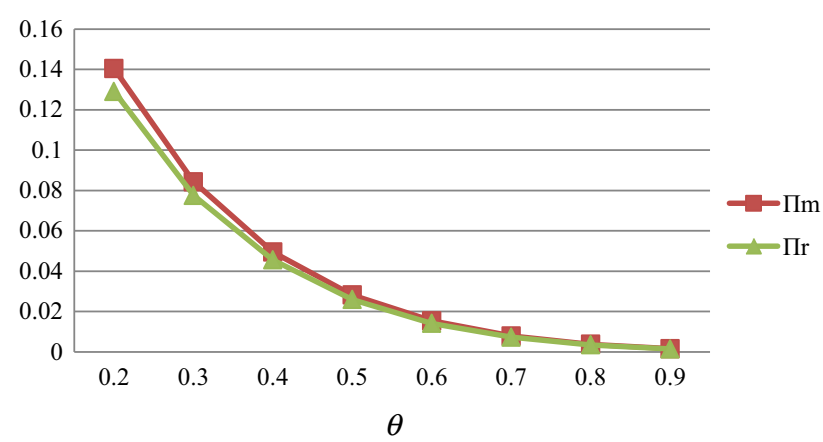

Fig. 1 Changes of manufacturer's and retailer's profit respect to the changes of $\theta$ in SM model

To analyze the manufacturer's and the retailer's decision variables in SR game structure, we use another example with $d_{1}=6, d_{2}=3, k_{m}=0.7, k_{r}=0.4$ and $\beta=0.04$.

\section{Conclusions}

In this paper, cooperative advertising models are developed for complementary products. Our models consist of SM and SR games in a two-echelon supply chain. In this model, the demand of each product is influenced by its own price, the price of the other good and advertising expenditures. The results showed that manufacturer's and retailer's profit decrease when the complementary degree between two products increases. When the complementary degree between products is large, it is better for the members to choose a strategy that increases their profit. They can offer different price discounts to their customers or choose bundling strategy. This work can be extended under several directions such as considering two retailers or two manufacturers, several market segments, and considering leakage between the markets.

Open Access This article is distributed under the terms of the Creative Commons Attribution License which permits any use,
Table 3 Influence of $\theta$ on variables in SR game

\begin{tabular}{llllll}
\hline$\theta$ & 0.2 & 0.3 & 0.4 & 0.5 & 0.6 \\
\hline$w_{1}^{*}$ & 9.7083 & 9.3076 & 8.9642 & 8.6666 & 8.4062 \\
$w_{2}^{*}$ & 7.4583 & 7.0576 & 6.7142 & 6.4166 & 6.1562 \\
$p_{1}^{*}$ & 13.4166 & 12.6153 & 11.9285 & 11.3333 & 10.812 \\
$p_{2}^{*}$ & 11.9166 & 11.1153 & 10.4285 & 9.8333 & 9.3125 \\
$q_{r}^{*}$ & 0.0248 & 0.0282 & 0.0318 & 0.0358 & 0.0402 \\
$q_{m}^{*}$ & 0.2575 & 0.2269 & 0.2009 & 0.1785 & 0.1591 \\
$t^{*}$ & 0 & 0 & 0 & 0 & 0 \\
$\pi_{m}^{*}$ & 1.0890 & 0.9095 & 0.7663 & 0.6505 & 0.5559 \\
$\pi_{r}^{*}$ & 1.3217 & 1.1082 & 0.9353 & 0.7932 & 0.6748 \\
\hline & & & & &
\end{tabular}

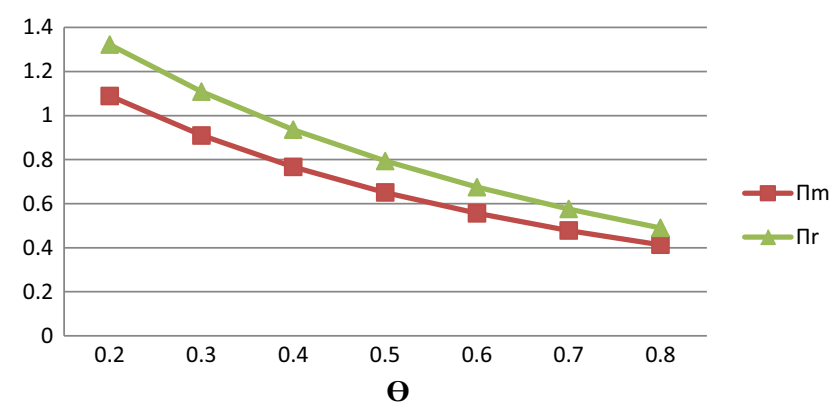

Fig. 2 Changes of manufacturer and retailer profit respect to the changes of $\theta$ in SR model

distribution, and reproduction in any medium, provided the original author(s) and the source are credited.

\section{Appendix A: Deriving the optimal values of SM model}

By substituting Eqs. (13), (14) and (15) into the manufacturer's profit function, we have:

$$
\begin{aligned}
\operatorname{Max} \pi_{m}= & {\left[\left(\frac{k_{r}^{2}}{8 \beta(1-t)(1+\theta)}\right)\right.} \\
& \times\left[\begin{array}{c}
\left(1-\beta w_{1}(1+\theta)\right)\left(1-\beta w_{1}-\beta w_{2} \theta\right)+ \\
\left(1-\beta w_{2}(1+\theta)\right)\left(1-\beta w_{2}-\beta w_{1} \theta\right)
\end{array}\right] \\
& \left.+k_{m} \sqrt{q_{m}}\right] \times\left[\left(w_{1}-d_{1}\right)\left(\frac{1-\beta w_{1}-\beta w_{2} \theta}{2}\right)\right. \\
& \left.+\left(w_{2}-d_{2}\right)\left(\frac{1-\beta w_{2}-\beta w_{1} \theta}{2}\right)\right] \\
& -t\left(\frac{k_{r}}{8 \beta(1+\theta)(1-t)}\right)^{2} \\
& \times\left[\left(1-\beta w_{1}(1+\theta)\right)\left(1-\beta w_{1}-\beta w_{2} \theta\right)\right. \\
& \left.+\left(1-\beta w_{2}(1+\theta)\right)\left(1-\beta w_{2}-\beta w_{1} \theta\right)\right]^{2}-q_{m}
\end{aligned}
$$


Let us introduce $z_{1}, z_{2}$ and $u$ and substitute into Eq. (A1) as follows:

$$
\begin{aligned}
z_{1}= & {\left[\left(1-\beta w_{1}(1+\theta)\right)\left(1-\beta w_{1}-\beta w_{2} \theta\right)\right.} \\
& \left.+\left(1-\beta w_{2}(1+\theta)\right)\left(1-\beta w_{2}-\beta w_{1} \theta\right)\right] \\
z_{2}= & \frac{1}{2}\left[\left(w_{1}-d_{1}\right)\left(1-\beta w_{1}-\beta w_{2} \theta\right)\right. \\
& \left.+\left(w_{2}-d_{2}\right)\left(1-\beta w_{2}-\beta w_{1} \theta\right)\right] \\
u= & \frac{k_{r}}{8 \beta(1-t)(1+\theta)} \\
\operatorname{Max} & \pi_{m}=\left[k_{r} u z_{1}+k_{m} \sqrt{q_{m}}\right] \times z_{2}-t u^{2} z_{1}^{2}-q_{m}
\end{aligned}
$$

By setting $\partial \pi_{m} / \partial q_{m}$ and $\partial \pi_{m} / \partial t$ equal to zero, after some simplifications we have:

$$
\begin{aligned}
q_{m}= & \left(\frac{k_{m}^{2}}{16}\right)\left[\left(w_{1}-d_{1}\right)\left(1-\beta w_{1}-\beta w_{2} \theta\right)\right. \\
& \left.+\left(w_{2}-d_{2}\right)\left(1-\beta w_{2}-\beta w_{1} \theta\right)\right]^{2} \\
t= & \left(\frac{z_{2}(8 \beta(1+\theta))-z_{1}}{z_{2}(8 \beta(1+\theta))+z_{1}}\right)
\end{aligned}
$$

By setting $\partial \pi_{m} / \partial w_{1}$ and $\partial \pi_{m} / \partial w_{2}$ equal to zero and substituting $q_{m}$ and $t$, we can obtain Eqs. (17) and (18). Note that when we substitute the optimal value of $w_{1}$ and $w_{2}$ into $z_{1}$ and $z_{2}$, we obtain $z_{1}^{*}$ and $z_{2}^{*}$.

\section{Appendix B: Deriving the Optimal values of SM model}

Manufacturer profit function is as follows:

$$
\begin{aligned}
\pi_{m}= & {\left[k_{r} \sqrt{a}+k_{m} \sqrt{A}\right]\left[\left(w_{1}-d_{1}\right)\left(1-\beta p_{1}-\beta \theta p_{2}\right)\right.} \\
& \left.+\left(w_{2}-d_{2}\right)\left(1-\beta p_{2}-\beta \theta p_{1}\right)\right]-t q_{r}-q_{m}
\end{aligned}
$$

St: $0 \leq t \leq 1,0<A, \quad w_{1}<p_{1}$ and $w_{2}<p_{2}$

By substituting Eqs. (24) and (25) into the manufacturer problem, after simplification we have:

$$
\begin{aligned}
\pi_{m}= & {\left[k_{r} \sqrt{a}+k_{m} \sqrt{A}\right] \times\left[( w _ { 1 } - d _ { 1 } ) \left(1-\beta 2 w_{1}\right.\right.} \\
& \left.\left.+\beta d_{1}-\beta \theta 2 w_{2}+\beta \theta d_{2}\right)\right]\left(w_{1}-d_{1}\right)\left(1-\beta 2 w_{1}+\beta d_{1}\right. \\
& \left.-\beta \theta 2 w_{2}+\beta \theta d_{2}\right)+\left(w_{2}-d_{2}\right)\left(1-\beta 2 w_{2}\right. \\
& \left.+\beta d_{2}-\beta \theta 2 w_{1}-\beta \theta d_{1}\right)-t q_{r}-q_{m}
\end{aligned}
$$

By solving the manufacturer problem, one can obtain:

$$
\begin{aligned}
\frac{\delta \pi_{m}}{\delta w_{1}}=0 \Rightarrow & \left(1-\beta 2 w_{1}+\beta d_{1}-\beta \theta 2 w_{2}+\beta \theta d_{2}\right) \\
& +(-2 \beta)\left(w_{1}-d_{1}\right)-2 \beta \theta\left(w_{2}-d_{2}\right)=0 \\
\Rightarrow & w_{1}=\frac{1-4 \beta \theta w_{2}+3 \beta d_{1}+3 \beta \theta d_{2}}{4 \beta}
\end{aligned}
$$

$$
\begin{aligned}
\frac{\delta \pi_{m}}{\delta w_{2}}=0 & \Rightarrow\left(1-\beta 2 w_{2}+\beta d_{2}-\beta \theta 2 w_{1}+\beta \theta d_{1}\right) \\
& +(-2 \beta)\left(w_{2}-d_{2}\right)-2 \beta \theta\left(w_{1}-d_{1}\right)=0 \\
& \Rightarrow w_{2}=\frac{1-4 \beta \theta w_{1}+3 \beta d_{2}+3 \beta \theta d_{1}}{4 \beta}
\end{aligned}
$$

\section{References}

Aust G, Buscher U (2012) Vertical cooperative advertising and pricing decisions in a manufacturer-retailer supply chains: A game theory approach. Eur J Oper Res 223:473-482

Gabszewicz J, Sonnac N, Wauthy X (2001) On price competition with complementary goods. Econ Lett 70:431-437

Gaski JF (1984) The theory of power and conflict in channels of distribution. J Mark 15:107-111

Hosseini Z, Ghasemy-Yaghin R, Esmaeili M (2013) A multiple objective approach for joint ordering and pricing planning problem with stochastic lead times. J Ind Eng Int 9:29

Huang Z, Li SX (2001) Co-op advertising models in a manufacturerretailer supply chains: a game theory approach. Eur J Oper Res 135:527-544

Huang Z, Li SX, Mahajan V (2002) An analysis of manufacturerretailer supply chain coordination in cooperative advertising. Decis Sci 33:469-494

Jørgensen S, Zaccour G (1999) Equilibrium pricing and advertising strategies in a marketing channel. J Optim Theory Appl 102:111-125

Jørgensen S, Sigue S, Zaccour G (2000) Dynamic cooperative advertising in a channel. J Retail 76:71-92

Munson CL, Rosenblatt MJ (2001) Coordinating a three-level supply chain with quantity discounts. IIE Trans 33:371-384

SeyedEsfahani MM, Biazaran M, Gharakhani M (2011) A game theory approach to coordinate pricing and vertical co-op advertising in a manufacturer-retailer supply chains. Eur J Oper Res 211:263-273

Szmerekovsky J, Zhang J (2009) Pricing and two-tier advertising with one manufacturer and one retailer. Eur J Oper Res 192:904-917

Taleizadeh AA, Noori-Daryan M (2015) Pricing, Manufacturing and inventory policies for raw material in a three-level supply chain. Int J Syst Sci, In Press

Taleizadeh AA, Nouri-Dariyan M, Cárdenas-Barrón LE (2015a) Joint optimization of price, replenishment frequency, replenishment cycle and production rate in vendor managed inventory system with deteriorating items. Int J Prod Econ 159:285-295

Taleizadeh AA, Noori-Daryan M, Tavakkoli-moghadam R (2015b) Pricing and ordering decisions in a supply chain with imperfect quality items and inspection under a buyback contract. Int J Prod Res, In Press

Wei J, Zhao J, Li Y (2013) Pricing decisions for complementary products with firms' different market powers. Eur J Oper Res 224:507-519

Xie J, Neyret A (2009) Co-op advertising and pricing models in manufacturer-retailer supply chains. Comput Ind Eng $56: 1375-1385$

Xie J, Wei J (2009) Coordinating advertising and pricing in a manufacturer-retailer channel. Eur J Oper Res 197:785-791

Yan R, Bandyopadhyay S (2011) The profit benefits of bundle pricing of complementary products. J Retail Consum Servs 18:355-361 
Yan R, Myers C, Wang J, Ghose S (2014) Bundling products to success: The influence of complementarity and advertising. J Retail Consum Serv 21:48-53

Yue J, Austin J, Wang M, Huang Z (2006a) Coordination of cooperative advertising in a two-level supply chain when manufacturer offers discount. Eur J Oper Res 168:65-85
Yue X, Mukhopadhyay S, Zhu X (2006b) A Bertrand model of pricing of complementary goods under information asymmetry. J Bus Res 59:1182-1192 